\title{
Detection of mesenchymal stem cells senescence by prelamin $A$ accumulation at the nuclear level
}

\author{
Chiara Bellotti ${ }^{1,2^{*}} \mathbb{D}$, Cristina Capanni $i^{3,4}$, Giovanna Lattanzi ${ }^{3,4}$, Davide Donati ${ }^{1,2}$, Enrico Lucarelli ${ }^{1}$ \\ and Serena Duchi $i^{1,2}$
}

\begin{abstract}
Background: Human mesenchymal stem cells (MSC), during in vitro expansion, undergo a progressive loss of proliferative potential that leads to the senescent state, associated with a reduction of their "medicinal" properties. This may hampers their efficacy in the treatment of injured tissues. Quality controls on MSC-based cell therapy products should include an assessment of the senescent state. However, a reliable and specific marker is still missing. From studies on lamin-associated disorders, has emerged the correlation between defective lamin A maturation and cellular senescence.
\end{abstract}

Findings: Primary cultured hMSC lines $(n=3)$, were analyzed by immunostaining at different life-span stages for the accumulation of prelamin A, along with other markers of cellular senescence. During culture, cells at the last stage of their life span displayed evident signs of senescence consistent with the positivity of SA- $\beta$-gal staining. We also observed a significant increase of prelamin A positive cells. Furthermore, we verified that the cells marked by prelamin A were also positive for p2 $1^{\text {Waf1 }}$ while negative for Ki67.

Conclusions: Overall data support that the detection of prelamin A identifies senescent MSC, providing an easy and reliable tool to be use alone or in combination with known senescence markers to screen MSC before their use in clinical applications.

Keywords: Mesenchymal stem cells, Lamin A, Prelamin A, Senescence, Cell- and tissue-based therapy

\section{Background}

Human mesenchymal stem cells (MSC) have raised high hopes in various therapeutic applications and their use is currently tested in about 500 clinical trials (www. clinicaltrials.gov). For many clinical approaches these cells are usually expanded in vitro prior to their utilization. However, MSC can undergo only a limited number of cell divisions under standard culture conditions, and it has been demonstrated that during in vitro proliferation they suffer a progressive and continuous process of aging (Wagner et al. 2008) that affects the proliferation and

\footnotetext{
*Correspondence: chiara.bellotti@ior.it

${ }^{1}$ Osteoarticular Regeneration Laboratory, 3rd Orthopaedic and Traumatologic Clinic Prevalently Oncologic, Rizzoli Orthopaedic Institute, via di Barbiano 1/10, Bologna 40036, Italy

Full list of author information is available at the end of the article
}

multilineage differentiation potential (Banfi et al. 2000; Kim et al. 2010), the immunomodulatory properties (Sepúlveda et al. 2014), the secretory profile (Coppe et al. 2010), the gene expression profile, and the epigenetic signature (Schellenberg et al. 2011; Yoo et al. 2013). These changes may impact the efficacy of MSC to treat injured tissues. For this reason, it is generally recommended in clinical applications to use MSC that have been cultured only for a restricted amount of time, fixing a threshold that limits their number of passages or population doublings (de Girolamo et al. 2013; Wuchter et al. 2014).

Cellular senescence is a complex process, which manifests with different, multifaceted phenotypes depending on the species, the cell type, and the senescence-inducing stimulus (Kosar et al. 2011). This lack of a unique signature implies the necessity for a mindful selection of the 
proper marker or panel of markers when assessing the senescence status of in vitro cultured MSC. The detection of SA- $\beta$-gal activity is the most common assay for the evaluation of cell senescence due to the availability of well-established protocols and commercial kits, and the abundant bibliography reporting its use. However the limitations of this marker are universally recognized (Crowe et al. 2014; Debacq-Chainiaux et al. 2008; Wagner et al. 2009). Other markers of senescence, opportunely reviewed by de Jesus (de Jesus and Blasco 2012), such as telomere shortening, Senescence-Associated Heterochromatin Foci (SAHF), Promyelocytic Leukemia Protein Nuclear Bodies, and Senescence-associated secretory phenotype (SASP) have been proposed over years. Recently, new approaches specifically focused on MSC have been suggested. Shibata et al. explored the expression of the $\mathrm{p} 16^{\mathrm{INK} 4 \mathrm{~A}}$ gene, suggesting its methylation state could be monitored as a surveillance against the transformation of MSC during culture (Shibata et al. 2007). Koch et al. proposed instead the use of the DNAmethylation changes observed at specific CpG sites in MSC and fibroblast to track the state of cellular senescence (Koch et al. 2012).

We moved from the observation of the phenomena that influence or lead to aging in humans, in order to identify a reliable and specific marker of the senescence phenotype of MSC.

In this regard, alteration of the nuclear lamina is known as one of the cellular changes observed in physiological aging (Vlcek and Foisner 2007; Lattanzi et al. 2014). Of particular interest is the $L M N A$ gene that encodes two components of the nuclear envelope: lamin $\mathrm{A}$ and $\mathrm{C}$. The maturation of lamin $\mathrm{A}$ is an elaborate process which involves several consecutive steps including: farnesylation, the proteolytic cleavage of three $\mathrm{N}$-terminal amino acids, the carboxymethylation and the final removal of additional fifteen $\mathrm{N}$-terminal amino acids including the farnesyl group. The final step is exclusively catalyzed by the zinc-metallopeptidase ZMPSTE24 encoded by the $F A C E-1$ gene. Mutations affecting different steps or actors of the maturation process, which elicits the accumulation of wild-type or mutated prelamin A, are associated with progeroid laminopathies or lipodystrophy (Broers and Ramaekers 2006; Davies et al. 2011). These diseases, including the Hutchinson-Gilford progeria syndrome that is characterized by premature aging, mainly affect tissues of mesenchymal origin, suggesting a link between prelamin A and MSC senescence.

The existence of this correlation was supported by the work of Scaffidi and Misteli. Their results demonstrated that the accumulation of wild type or mutant lamin A by means of expression vectors or drugs leads to an accelerated aging of human fibroblast and immortalized
MSC (Scaffidi and Misteli 2008). Our goal was to verify this correlation the other way around, and so where replicative senescence of primary MSC culture leads to prelamin A accumulation. The presence of lamin A precursors in cells after their prolonged in vitro culture or in tissue specimens from aged donors was already observed by other investigators, but their analysis was focused on Vascular Smooth Muscle Cells (Ragnauth et al. 2010).

As far as we know, a general and robust detection analysis of lamin A precursor in MSC that have naturally exited the replicative cycle in normal culture conditions has never been reported. Therefore, in our work we used primary cultures of human MSC isolated from the bone marrow of healthy donors to investigate the presence of unprocessed lamin A precursor during early and late stages of in vitro cultures, with the ultimate scope of proposing a proper marker to detect senescent MSC.

\section{Methods}

Primary human MSC were obtained from 3 non-oncologic patients (aged 20, 26, 6) during routine orthopedic surgical procedures.

Cell isolation and expansion is described in the Additional file.

\section{Definition of early and late stages of in vitro MSC culture}

MSC were maintained in culture until they reached their maximal life span as evidenced by growth arrest (i.e. the cells failed to become confluent within 4 weeks of culture).

The number of population doublings (PD) for each passage was calculated using the formula: $\log _{2}\left(\mathrm{~N}_{1} / \mathrm{N}_{0}\right)$, where $\mathrm{N}_{0}$ is the number of cells seeded and $\mathrm{N}_{1}$ is the number of cells harvested at the end of the passage. Cumulative population doublings (CPD) were calculated as the sum of PDs over passages.

CPD curves were normalized with GraphPad Prism 6 Software to set the maximum CPD value as the $100 \%$ of the cell line life-span. Early and late life-span stages were then identified by graphical interception on the CPD curve tracing horizontal lines at y coordinates equal to 50 and $80 \%$ (Stenderup et al. 2003). Experimental observations were performed on cell samples at passages comprised in the "early stage" (life-span $<50 \%$ ) or "late stage" (life-span $>80 \%$ ).

\section{Senescence associated $\beta$-galactosidase assay}

SA- $\beta$-gal activity was detected with a senescent cell staining kit (Sigma Aldrich, St. Louis, MO, USA) according to the manufacturer's instructions. Briefly, the $40 \mathrm{mg} /$ $\mathrm{ml}$ stock solution of 5 -bromo-4-chloro-3-indolyl $\beta$-dgalactopyranoside (X-gal) was prepared in the laboratory by dissolving the X-gal powder (Sigma Aldrich, USA) in 
$\mathrm{N}, \mathrm{N}$-dimethylformamide. Once prepared, it was stored at $-20{ }^{\circ} \mathrm{C}$ and used within a month, to ensure the accuracy of the assay.

Cells were seeded in a 24-well plate and cultivated until $60 \%$ confluence. Plates were washed with PBS, fixed for 5 min at room temperature, and incubated at $37^{\circ} \mathrm{C}$ overnight in a dry incubator with freshly prepared $1 \mathrm{mg} /$ $\mathrm{ml} \mathrm{X-gal} \mathrm{buffered} \mathrm{solution.} \mathrm{Cell} \mathrm{nuclei} \mathrm{were} \mathrm{counter-}$ stained with $5 \mu \mathrm{g} / \mathrm{ml}$ Hoechst 33342 (Life Technologies, Eugene, OR, USA). Microphotographs of a minimum of six random fields for each sample were taken using an epifluorescence microscope (Nikon Eclipse TE2000-U, Amsterdam, Netherland) equipped with a Nikon DS-Vi1U3 CCD color digital camera. Brightfield and fluorescent images were merged with NIS-D software (Nikon, Amsterdam, Netherlands) to count total and $\beta$-gal positive cells.

\section{Prelamin A detection and immunostaining}

MSC grown on coverslips were fixed in cold methanol at $-20{ }^{\circ} \mathrm{C}$ for $7 \mathrm{~min}$. Samples were incubated with PBS containing $4 \% \mathrm{BSA}$ to saturate non-specific binding and incubated overnight at $4{ }^{\circ} \mathrm{C}$ with anti-prelamin A antibody (Santa Cruz Sc-6214) diluted at 1:100. Coverslips were then washed several times in PBS and incubated $1 \mathrm{~h}$ at RT with donkey anti-goat secondary antibody (Santa Cruz Sc-3853) diluted 1:100. After washes with PBS, the nuclei were counterstained with 4,6-diamino-2-phenylindole (DAPI). The slides were mounted with an anti-fade reagent in glycerol and observed. To measure the percentage of prelamin A positive cells, microphotographs of a minimum of 6 random fields were taken for each sample and prelamin A positive cells were manually counted. Imaging was performed using a laser-scanning motorized confocal system (Nikon A1R, Nikon, Amsterdam, Netherlands) equipped with an Eclipse Ti-E inverted microscope and four laser lines $(405,488,561$, and $638 \mathrm{~nm})$. A Plan Apo VC 60x/1.4NA Oil DIC N2 objective lens was used. Images were processed using NIS-Elements AR 4.10.01 software (Nikon, Amsterdam, Netherlands).

As a positive control, accumulation of prelamin A was obtained using $25 \mu \mathrm{M}$ mevinolin (M2147, Sigma) in complete growth medium for $18 \mathrm{~h}$. Mevinolin is an isoprenoid synthesis inhibitor that causes inhibition of hydroxymethyl-glutaryl-synthase implicated in the farnesyl pathway. This compound elicits an accumulation of non-farnesylated (unprocessed) prelamin A, as reported in several studies (Mattioli et al. 2008).

To observe prelamin A nuclear distribution, confocal imaging was performed using the $488 \mathrm{~nm}$ laser line, and laser power was adjusted to minimize photobleaching. A Plan Apo 100x/1.4NA Oil DIC H objective lens was used. Zoom and field-of-view dimensions were adjusted to give a resolution of $1024 \times 1024$. Z-slices were acquired every $0.175 \mu \mathrm{m}$ for a total of 31 steps. NIS elements software permits 3D rendering of the $Z$-slices.

Two double immunostainings of prelamin A with p21 or Ki67 were performed. The rabbit polyclonal anti-Ki67 antibody (Santa Cruz Sc-15402) was diluted 1:100 and incubated overnight at $4{ }^{\circ} \mathrm{C}$ while the rabbit monoclonal anti-p21antibody [Pierce p21 Waf1/Cip1 Antibody (R.229.6)] was diluted 1:300 and incubated overnight at $4{ }^{\circ} \mathrm{C}$. Immunofluorescence microscopy was performed using a Nikon E600 epifluorescence microscope and a Nikon oil-immersion objective [100 $\times$ magnification, 1,3 NA (numerical aperture)]. Photographs were taken using a Nikon digital camera (DXm) and NIS-Element AR software.

\section{Results and discussion}

In the production of MSC-based cell therapy products, safety and potency evaluations are mandatory, and among other aspects, the senescent state of culture should be assessed.

Though alternatives to the classical SA- $\beta$-gal staining have been proposed (Shibata et al. 2007; Righolt et al. 2011; Koch et al. 2012) we believe an optimal marker for the identification of senescent MSC is not yet available. A correlation between accumulation of prelamin A and cellular aging has been already postulated (Scaffidi and Misteli 2008; Yu and Kang 2013; Yu et al. 2013). However, the main findings of the previous studies were mainly obtained through an induced accumulation of prelamin A with expression vectors or drugs. Our goal was to demonstrate that the spontaneous accumulation of prelamin A occurs in human MSC under a condition of replicative senescence induced by the prolonged in vitro culture.

MSC samples were isolated from the bone marrow of three healthy donors and maintained under standard culture condition until they reached replicative senescence in culture. As expected, when expanded in vitro each of them presented a distinctive growth curve and progression toward the Hayflick limit (Shay and Wright 2000) (Fig. 1a). Even if cells are isolated and cultured under the same conditions, the number of passages is evidently an unreliable indicator of cellular aging. Observations were therefore planned after the analysis of the growth curves of each cell line both at early and late stages (Fig. 1b), as described in the Materials and Methods section. The comparison of cells belonging to the same life-span stage attenuates the inter-donor variability and allows the observation of a coherent range of modifications such as a gradual change from a fibroblastic-like spindle shape to a large widespread morphology (Fig. 1c) and a reduced proliferation potential in the late stage (Fig. 1d). These 


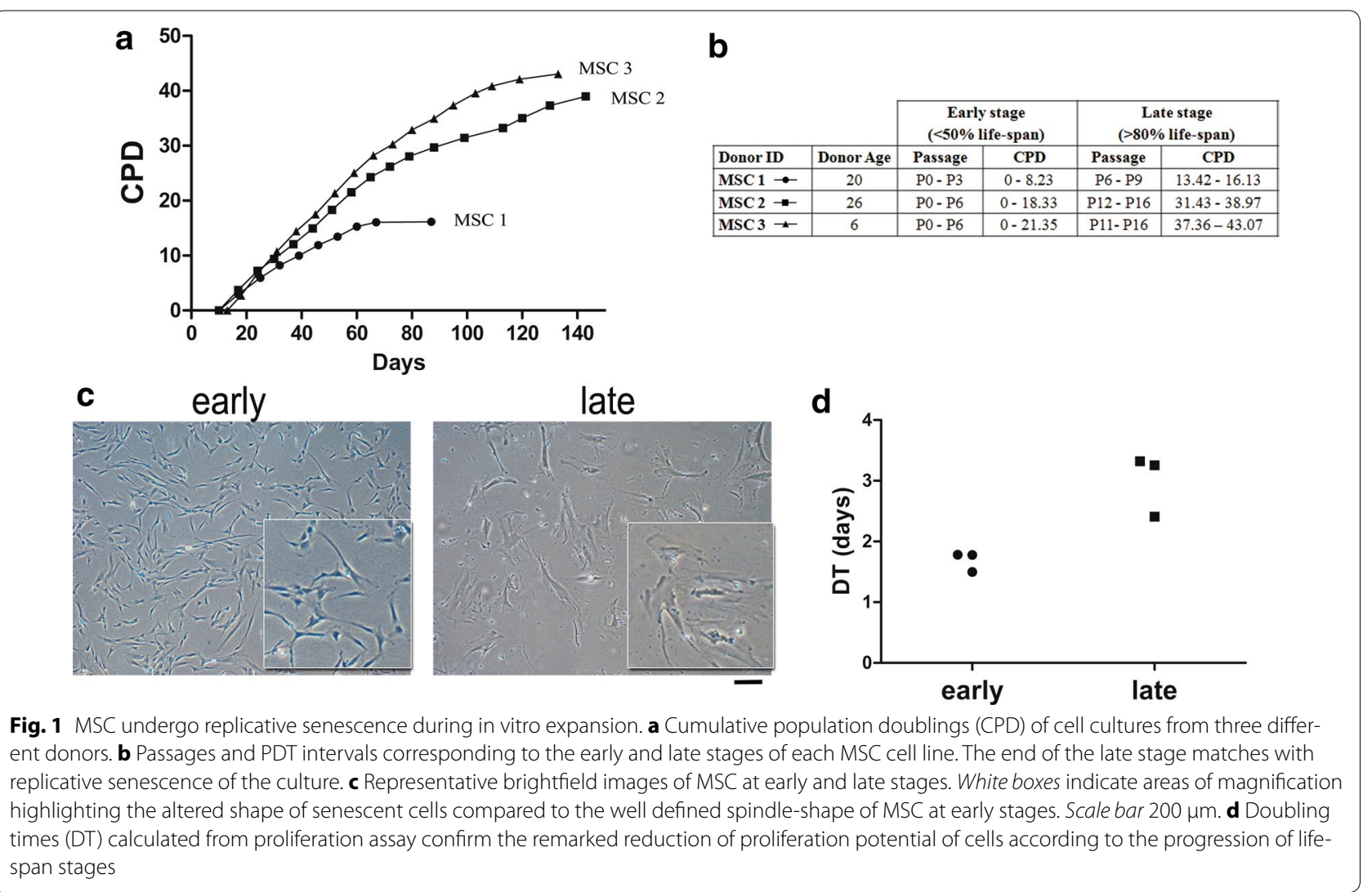

morphological changes are associated with cytoskeleton alteration. In fact, $\beta$-tubulin and Vimentin immunostaining analyses (Additional file 1: Fig. S1A, B), performed at early and late stages demonstrated the altered polarization and disruption of cytoskeletal microtubules filaments of senescent cells (Geißler et al. 2012).

The senescent status of the cell cultures at late stages was confirmed by the SA- $\beta$-gal assay that revealed an increased activity of the lysosomal $\beta$-D-galactosidase at the suboptimal $\mathrm{pH}$ of 6.0 , compared to the cells at early stage (Fig. 2a). Similarly, the specific immunostaining for the full-length form of lamin A precursor (with an antibody directed to the $\mathrm{C}$-terminal residue of the unprocessed protein) showed a higher number of cells positive for prelamin A at late stages (Fig. 2b). Confocal microscopy performed at high magnification confirmed a nucleoplasmic and rim localization of prelamin A together with an abnormal nuclear morphology (Fig. 2c, $\left.c^{\prime}\right)$. From the single $z$-stacks representative of different planes along the nucleus (Fig. 2c'), multiple invaginations of the nuclear membrane are evident. Interestingly, the observation of an increased incidence of wrinkled nuclei in senescent cells was exploited by Righolt et al. to elaborate an imaging method that quantifies the intensity and curvature of the nuclear lamina to identify abnormal cells during aging, in vitro proliferation, and in lamina disorders (Righolt et al. 2011).

The manual count of the percentage of SA- $\beta$-gal or prelamin A positive cells over the total performed on the 3 MSC lines confirmed the qualitative results. The percentage of prelamin A-positive cells increases dramatically from early to late stages (from 6 to $59 \%$ ), with a trend similar to the one observed for the SA- $\beta$-gal staining (Fig. 2d). Though the trends were similar we can observe that the results from prelamin A staining indicate a slightly higher percentage of positive cells than for SA- $\beta$-gal assay both at early and late stage. The number of observations is insufficient to operate a statistical comparison, but the observed difference could indicate that the accumulation of prelamin $\mathrm{A}$ is a more sensitive marker to reveal senescent cells even at earlier stages. The detection of prelamin A positive cells at early stage is not entirely unexpected, as primary MSC culture are known to be composed by an heterogeneous population that might harbor among the active proliferating cells, resting, terminally differentiated or senescent cells (Sherley 2002; Whitfield et al. 2013).

In addition, the variability among MSC lines observed for SA- $\beta$-gal counting despite we performed our analyses at stages selected to reduce the inter-donor variability 

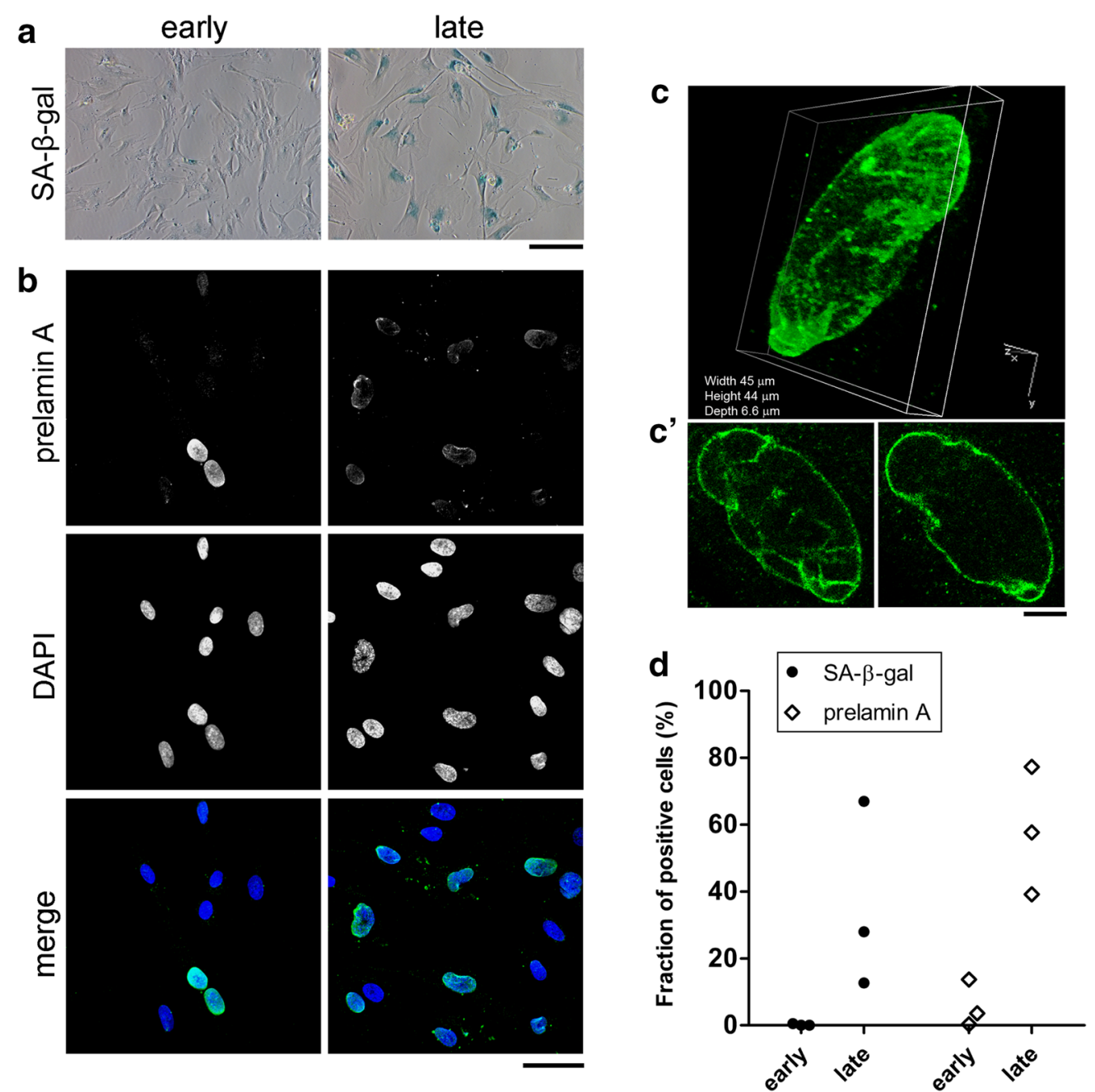

Fig. 2 MSC under replicative senescence accumulate prelamin A. a Representative microphotograph of SA- $\beta$-gal assay performed on early and late stage cells. Blue staining indicates the presence of $\beta$-galactosidase activity in senescent cells. Scale bar $200 \mu \mathrm{m}$. b Cells at early and late stages were fixed and immunostained with a specific antibody against prelamin A and counterstained with DAPI. Scale bar 50 $\mu$ m. c 3D digital rendering of Z-slices confocal images of a representative prelamin A positive cell at late stage. $\mathbf{c}^{\prime}$ Single slice images of different Z-stacks illustrating the distribution of prelamin A, from surface (left panel) and from middle plane (right panel) point of views. Scale bar $10 \mu \mathrm{m}$. d Quantification of $\beta$-gal positive and prelamin A positive cells at early and late stages. Blue stained cells and Hoechst stained nuclei were counted in a minimum of six random fields to report the percentage $\beta$-gal positive cells at early and late stages. To count prelamin A positive and total cell numbers a minimum of six random fields was checked at early and late stages. Data are expressed as percentage of positive cells respect to the total cell count

(Fig. 1), is reduced in the prelamin A scoring, making the latest a more steady marker.

The discordant results from the two markers might also be a consequence of the limitations and technical pitfalls inherent to the SA- $\beta$-gal assay caused by the limited specificity and instability of $\beta$-gal substrate, as already outlined by other investigators (Yang and $\mathrm{Hu}$ 2005; Lee et al. 2006; Debacq-Chainiaux et al. 2009). In this regard, the assessment of prelamin A accumulation can be performed with an easy available positive control, in order to check the technical quality of the immunostaining. The positive control for prelamin A consists in cells treated with mevinolin, a drug known to inhibit farnesyl production and to cause accumulation of unprocessed prelamin A (Lattanzi et al. 2014) (Additional file 1: Fig. S2). As expected from previous studies (Caron et al. 2007), treatment with mevinolin did not induce SA- $\beta$-gal expression (Additional file 1: Fig. S2). This is in agreement with our data showing that cells from centenarian individuals spontaneously accumulate prelamin A and display a more efficient response to oxidative stress-induced DNA damage (Lattanzi et al. 
2014). These data support the view that prelamin A accumulation does not induce senescence per se, but it is triggered in response to stress stimuli in the attempt to counteract geroconversion. In particular, we observed in human fibroblast that the oxidative and replicative stress affect prelamin A processing, through the reduction of ZMPSTE24 expression (Lattanzi et al. 2014) and it could be speculated that a similar mechanism is involved in prelamin A accumulation observed in senescent MSC.

Biochemical evaluation of prelamin A was also performed by Western blotting analysis. Prelamin A, in accordance with results obtained by prelamin A immunofluorescence staining, is detectable at the predicted molecular weight in both senescent and mevinolintreated MSCs (Additional file 1: Fig. S3).

We further checked the expression of $\mathrm{p} 21^{\text {Waf1 }}(C d k n 1 n$ gene), a nuclear protein that indicates senescence-associated cell-cycle arrest (Kong et al. 2011), and Ki67, a marker of proliferating cells expressed in all active phases of the cell cycle $\left(G_{1}, S, G_{2}\right)$. As hypothesized, cells positive for prelamin A showed p21 ${ }^{\text {Waf1 }}$ staining in nuclei and were negative for Ki67 (Fig. 3), confirming that prelamin A-labeled MSC underwent replicative senescence during in vitro culture.

Based on our observations, the nuclear accumulation of prelamin A identifies senescent cells in human MSC cultures.
It is worth noting that previous reports indicate that the accumulation of mutated forms or precursors of lamin A triggers a series of molecular changes that diminish the specific properties of the MSC such as the multilineage differentiation potential ( $\mathrm{Yu}$ et al. 2013; Malashicheva et al. 2015) and the capacity to promote the repair of injured tissues (Infante et al. 2014). Therefore, prelamin A accumulation can be therefore considered a candidate marker for the detection of senescent cells during MSC expansion and can be exploited to discard aged cells, characterized by low differentiation and regeneration capacity, and thus prevent their release for clinical purposes.

\section{Conclusions}

Despite the underlying molecular mechanisms are still unraveled, replicative senescence has evident consequences for cellular therapy. In this paper we have demonstrated that in human MSC cultured in vitro under standard growth condition, the detection of prelamin A identifies senescent MSC. Since the onset of senescence in a MSC culture is currently difficult to predict by the use of available markers, prelamin A staining could be successfully used to screen MSC populations before they are used for clinical applications alone or in combination with know and currently used senescence marker.

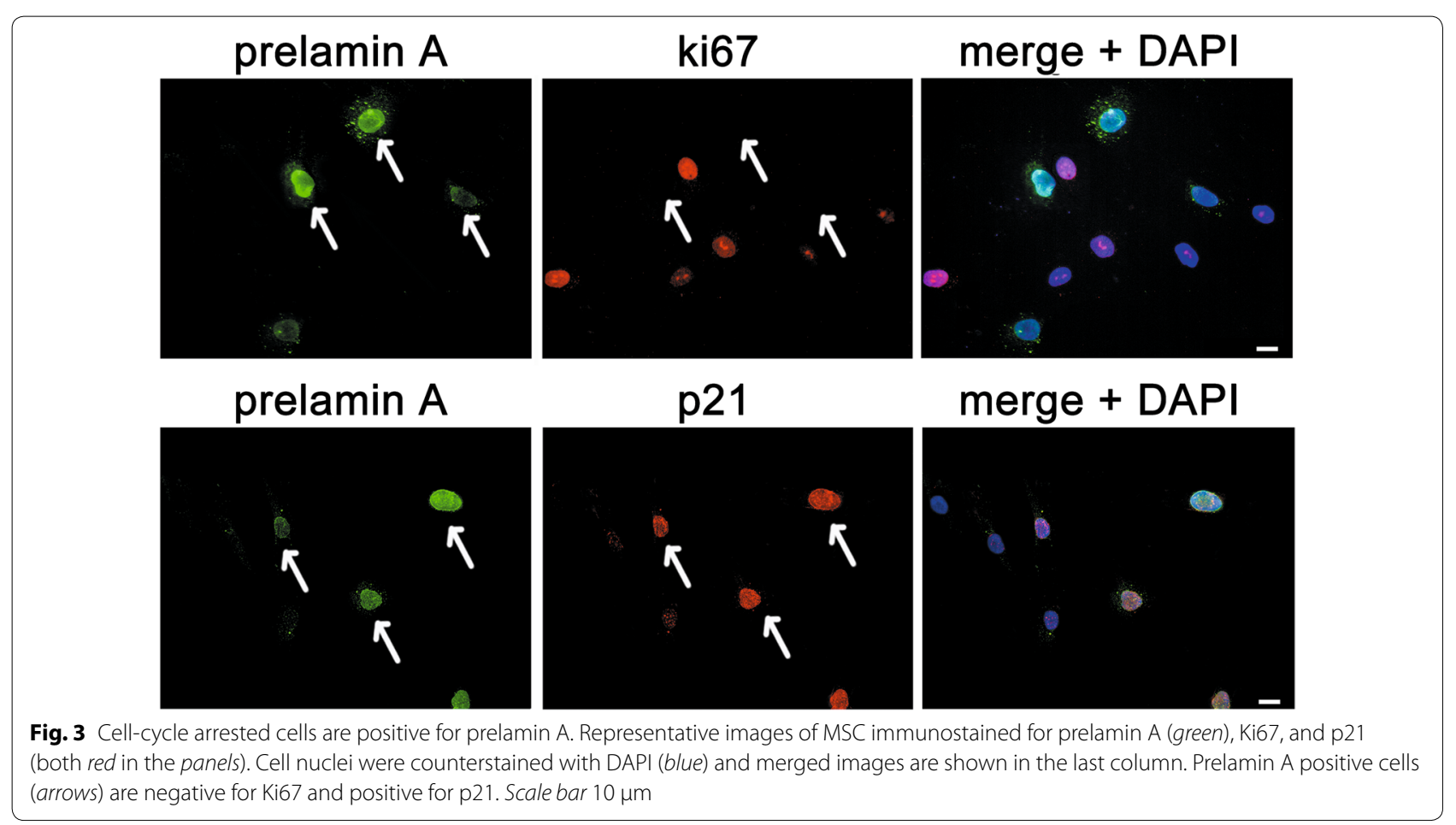




\section{Additional file}

Additional file 1. Supplementary data.

\section{Abbreviations}

SA- $\beta$-gal: senescence associated $\beta$-galactosidase; MSC: mesenchymal stem cells.

\section{Authors' contributions}

CB designed and performed the experiments, analysed the data and wrote the manuscript. CC formulated the initial hypothesis, gave conceptual contribution to the study, performed the experiments and revised the manuscript. GL provided financial support, gave conceptual contribution to the study and revised the manuscript. DD provided financial support and performed the harvest of bone-marrow samples. EL gave conceptual contribution to the study and revised the manuscript. SD designed and coordinated the study, performed the experiments, analysed the data and wrote the manuscript. All authors read and approved the final manuscript.

\section{Author details}

${ }^{1}$ Osteoarticular Regeneration Laboratory, 3rd Orthopaedic and Traumatologic Clinic Prevalently Oncologic, Rizzoli Orthopaedic Institute, via di Barbiano 1/10, Bologna 40036, Italy. ${ }^{2}$ Department of Biomedical and Neuromotor Sciences, University of Bologna, Bologna, Italy. ${ }^{3}$ Institute of Molecular Genetics - Unit of Bologna, CNR-National Research Council of Italy, Bologna, Italy. ${ }^{4}$ Laboratory of Musculoskeletal Cell Biology, Rizzoli Orthopaedic Institute, Bologna, Italy.

\section{Acknowledgements}

The authors would like to thank the staff of the Third Orthopaedics and Traumatology Clinic (IOR, Bologna), for providing the cells used in this work, and Miss Lauren DeMaria BSc (Columbia University NY) for editorial assistance and English revision of the manuscript. The research was supported by Progetto FIRB-Accordi di programma 2010 (cod.RBAP10447J) of Italian Ministry of Education Universities and Research and by "5 per mille" 2011/2012 funding from Italian Ministry of Health.

\section{Competing interests}

The authors declare that they have no competing interests.

\section{Research involving human participants}

The study was reviewed and approved by the local Ethical Committee (Approval Record No. 21621). Patients signed an informed consent before the donation of bone marrow.

Received: 11 February 2016 Accepted: 17 August 2016

Published online: 26 August 2016

\section{References}

Banfi A, Muraglia A, Dozin B et al (2000) Proliferation kinetics and differentiation potential of ex vivo expanded human bone marrow stromal cells: implications for their use in cell therapy. Exp Hematol 28:707-715

Broers J, Ramaekers F (2006) Nuclear lamins: laminopathies and their role in premature ageing. Physiol Rev 86:967-1008. doi:10.1152/ physrev.00047.2005

Caron M, Auclair M, Donadille B et al (2007) Human lipodystrophies linked to mutations in A-type lamins and to HIV protease inhibitor therapy are both associated with prelamin A accumulation, oxidative stress and premature cellular senescence. Cell Death Differ 14:1759-1767. doi:10.1038/ sj.cdd.4402197

Coppe J-P, Desprez P-Y, Krtolica A, Campisi J (2010) The senescence-associated secretory phenotype: the dark side of tumor suppression. Annu Rev Pathol 5:99-118. doi:10.1146/annurev-pathol-121808-102144

Crowe EP, Nacarelli T, Bitto A et al (2014) Detecting senescence: methods and approaches. In: Noguchi E, Gadaleta MC (eds) Cell cycle control. Springer, New York, NY, pp 425-445
Davies BS, Coffinier C, Yang SH et al (2011) Investigating the purpose of prelamin A processing. Nucleus 2:4-9. doi:10.4161/nucl.2.1.13723

de Girolamo L, Lucarelli E, Alessandri G et al (2013) Mesenchymal stem/stromal cells: a new cells as drugs" paradigm. Efficacy and critical aspects in cell therapy. Curr Pharm Des 19:2459-2473

de Jesus BB, Blasco MA (2012) Assessing cell and organ senescence biomarkers. Circ Res 111:97-109. doi:10.1161/CIRCRESAHA.111.247866

Debacq-Chainiaux F, Pascal T, Boilan E et al (2008) Screening of senescenceassociated genes with specific DNA array reveals the role of IGFBP-3 in premature senescence of human diploid fibroblasts. Free Radic Biol Med 44:1817-1832. doi:10.1016/j.freeradbiomed.2008.02.001

Debacq-Chainiaux F, Erusalimsky JD, Campisi J, Toussaint O (2009) Protocols to detect senescence-associated beta-galactosidase (SA-betagal) activity, a biomarker of senescent cells in culture and in vivo. Nat Protoc 4:1798-1806. doi:10.1038/nprot.2009.191

Geißler S, Textor M, Kühnisch J et al (2012) Functional comparison of chronological and in vitro aging: differential role of the cytoskeleton and mitochondria in mesenchymal stromal cells. PLoS ONE 7:e52700. doi:10.1371/ journal.pone.0052700

Infante A, Gago A, de Eguino GR et al (2014) Prelamin A accumulation and stress conditions induce impaired Oct- 1 activity and autophagy in prematurely aged human mesenchymal stem cell. Aging (Albany NY) 1:1-17

Kim J, Choi YS, Lim S et al (2010) Comparative analysis of the secretory proteome of human adipose stromal vascular fraction cells during adipogenesis. Proteomics 10:394-405. doi:10.1002/pmic.200900218

Koch CM, Joussen S, Schellenberg A et al (2012) Monitoring of cellular senescence by DNA-methylation at specific CpG sites. Aging Cell 11:366-369

Kong Y, Cui H, Ramkumar C, Zhang H (2011) Regulation of senescence in cancer and aging. J Aging Res 2011:963172. doi:10.4061/2011/963172

Kosar M, Bartkova J, Hubackova S et al (2011) Senescence-associated heterochromatin foci are dispensable for cellular senescence, occur in a cell type- and insult-dependent manner and follow expression of p16 ink4a Cell Cycle 10:457-468. doi:10.4161/cc.10.3.14707

Lattanzi G, Ortolani M, Columbaro M et al (2014) Lamins are rapamycin targets that impact human longevity: a study in centenarians. J Cell Sci 127:147-157. doi:10.1242/jcs.133983

Lee BY, Han JA, Im JS et al (2006) Senescence-associated $\beta$-galactosidase is lysosomal $\beta$-galactosidase. Aging Cell 5:187-195. doi:10.1111/j.1474-9726.2006.00199.x

Malashicheva A, Bogdanova M, Zabirnyk A et al (2015) Various lamin A/C mutations alter expression profile of mesenchymal stem cells in mutation specific manner. Mol Genet Metab. doi:10.1016/j. ymgme.2015.04.006

Mattioli E, Columbaro M, Capanni C et al (2008) Drugs affecting prelamin A processing: effects on heterochromatin organization. Exp Cell Res 314:453-462. doi:10.1016/j.yexcr.2007.11.012

Ragnauth CD, Warren DT, Liu Y et al (2010) Prelamin A acts to accelerate smooth muscle cell senescence and is a novel biomarker of human vascular aging. Circulation 121:2200-2210. doi:10.1161/ CIRCULATIONAHA.109.902056

Righolt CH, Van't Hoff MLR, Vermolen BJ et al (2011) Robust nuclear laminabased cell classification of aging and senescent cells. Aging (Albany NY) 3(1192-1201):1004

Scaffidi P, Misteli T (2008) Lamin A-dependent misregulation of adult stem cells associated with accelerated ageing. Nat Cell Biol 10:452-459. doi:10.1038/ncb1708

Schellenberg A, Lin Q, Schüler H et al (2011) Replicative senescence of mesenchymal stem cells causes DNA-methylation changes which correlate with repressive histone marks. Aging (Albany NY) 3:873-888

Sepúlveda JC, Tomé M, Fernández ME et al (2014) Cell senescence abrogates the therapeutic potential of human mesenchymal stem cells in the lethal endotoxemia model. Stem Cells. doi:10.1002/stem.1654

Shay JW, Wright WE (2000) Hayflick, his limit, and cellular ageing. Nat Rev Mol Cell Biol 1:72-76. doi:10.1038/35036093

Sherley JL (2002) Asymmetric cell kinetics genes: the key to expansion of adult stem cells in culture. Stem Cells 20:561-572. doi:10.1634/ stemcells.20-6-561

Shibata KR, Aoyama T, Shima Y et al (2007) Expression of the p16INK4A gene is associated closely with senescence of human mesenchymal stem cells and is potentially silenced by DNA methylation during in vitro expansion. Stem Cells 25:2371-2382. doi:10.1634/stemcells.2007-0225 
Stenderup K, Justesen J, Clausen C, Kassem M (2003) Aging is associated with decreased maximal life span and accelerated senescence of bone marrow stromal cells. Bone 33:919-926. doi:10.1016/j.bone.2003.07.005

Vlcek S, Foisner R (2007) Lamins and lamin-associated proteins in aging and disease. Curr Opin Cell Biol 19:298-304. doi:10.1016/j.ceb.2007.04.001

Wagner W, Horn P, Castoldi M et al (2008) Replicative senescence of mesenchymal stem cells: a continuous and organized process. PLOS ONE 3:e2213. doi:10.1371/journal.pone.0002213

Wagner W, Bork S, Horn P et al (2009) Aging and replicative senescence have related effects on human stem and progenitor cells. PLoS ONE 4:e5846

Whitfield MJ, Lee WCJ, Van Vliet KJ (2013) Onset of heterogeneity in cultureexpanded bone marrow stromal cells. Stem Cell Res 11:1365-1377. doi:10.1016/j.scr.2013.09.004

Wuchter P, Bieback K, Schrezenmeier H et al (2014) Standardization of Good Manufacturing Practice-compliant production of bone marrow-derived human mesenchymal stromal cells for immunotherapeutic applications. Cytotherapy 17:128-139. doi:10.1016/j.jcyt.2014.04.002
Yang NC, Hu ML (2005) The limitations and validities of senescence associated- $\beta$ - galactosidase activity as an aging marker for human foreskin fibroblast Hs68 cells. Exp Gerontol 40:813-819. doi:10.1016/j. exger.2005.07.011

Yoo JK, Choi S-J, Kim JK (2013) Expression profiles of subtracted mRNAs during cellular senescence in human mesenchymal stem cells derived from bone marrow. Exp Gerontol. doi:10.1016/j.exger.2013.02.022

Yu K-R, Kang K-S (2013) Aging-related genes in mesenchymal stem cells: a mini-review. Gerontology. doi:10.1159/000353857

Yu KR, Lee S, Jung JW et al (2013) MicroRNA-141-3p plays a role in human mesenchymal stem cell aging by directly targeting ZMPSTE24. J Cell Sci 126:5422-5431. doi:10.1242/jcs.133314

\section{Submit your manuscript to a SpringerOpen ${ }^{\odot}$ journal and benefit from:}

- Convenient online submission

- Rigorous peer review

- Immediate publication on acceptance

- Open access: articles freely available online

- High visibility within the field

- Retaining the copyright to your article 